\title{
Numerical Method for One-Dimensional Convection-diffusion Equation Using Radical Basis Functions
}

\author{
Su LD ${ }^{1,2 *}$, Jiang $Z W^{2}$ and Jiang TS $^{2 *}$
}

${ }^{1}$ North-Eastern Federal University, Belinskogo, Yakutsk, Russia

${ }^{2}$ Department of Mathematics, Linyi University, Linyi, P.R. China

\begin{abstract}
In this paper, the meshless method is employed for the numerical solution of the one-dimensional (1D) convection-diffusion equation based on radical basis functions (RBFs). Coupled with the time discretization and the collocation method, the proposed method is a truly meshless method which requires neither domain nor boundary discretization. The algorithm is very simple so it is very easy to implement. The results of numerical experiments are presented, and are compared with analytical solutions to confirm the good accuracy of the presented scheme.
\end{abstract}

Keywords: Meshless method; Radical basis function (RBF); Numerical solution; Convection-diffusion equation

\section{Introduction}

Whenever we consider mass transport of a dissolved species (solute species) or a component in a gas mixture, concentration gradients will cause diffusion. If there is bulk fluid motion, convection will also contribute to the flux of chemical species. Therefore, we are often interested in solving for the combined effect of both convection and diffusion.

The convection-diffusion equation is a combination of the diffusion and convection (advection) equations, and describes physical phenomena where particles, energy, or other physical quantities are transferred inside a physical system due to two processes: diffusion and convection [1]. $[2,3]$

The general convection-diffusion equation has the following form

$$
\frac{\partial u(\mathrm{x}, t)}{\partial t}=\nabla \cdot(D \nabla u(\mathrm{x}, t))-\nabla(v u(\mathrm{x}, t))+R(\mathrm{x}, t) .
$$

In the above equation, four terms represents transient, convection, diffusion and source term respectively. Where $u(x, t)$ is the variable of interest (species concentration for mass transfer, temperature for heat transfer), D is the diffusivity (also called diffusion coefficient), $V$ is the average velocity that the quantity is moving, $R(x, t)$ is source term represents capacity of internal sources, $\nabla$ represents gradient and $\nabla$. represents divergence.

This paper is devoted to the numerical computation of the onedimension

(1D) convection-diffusion equation:

$$
\mathrm{u}_{\mathrm{t}}(\mathrm{x}, \mathrm{t})+\alpha \mathrm{u}_{\mathrm{x}}(\mathrm{x}, \mathrm{t})+\beta \mathrm{u}(\mathrm{x}, \mathrm{t})=\varepsilon \mathrm{u}_{\mathrm{xx}}+\mathrm{f}(\mathrm{x}, \mathrm{t}), \mathrm{a} \leq \mathrm{x} \leq \mathrm{b}, 0 \leq \mathrm{t},
$$

With the initial conditions:

$$
u(x, 0)=h(x), a \leq x \leq b,
$$

And Dirichlet boundary conditions:

$$
u(a, t)=g_{0}(t), u(b, t)=g_{1}(t), 0 \leq \mathrm{t}
$$

Where $\alpha, \beta$ and $\varepsilon$ are known constant coefficients, $h(x)$ and $g_{i}(t)$ $(i=0,1)$ are known continuous functions.

Recently, much attention has been given to the development, analysis, and implementation of stable methods for the numerical solution of the convection-diffusion equations (see [4] and the reference therein). Jim Douglas, et al. [5] combine definite element and finite difference methods based on the method of characteristic for solving the convection-diffusion problems. Chen and Hon [6] consider the 2D and 3D Helmholtz and convection-diffusion equation using boundary knot method. The meshless local Petro-Galerkin method for convection-diffusion equation was considered in [7]. A new finite difference method described by Ram P. Manohar and John W. Stephenson [8].

In this article, we present a numerical scheme to solve the convection-diffusion equation using the collocation method with Radial Basis Function (RBF). The results of numerical experiments are presented, and are compared with analytical solutions to confirm the good accuracy of the presented scheme.

In last 25 years, the radial basis functions (RBFs) method is known as a powerful tool for scattered data interpolation problem. The use of RBFs as a meshless procedure for numerical solution of partial differential equations is based on the collocation scheme. Because of the collection technique, this method does not need to evaluate any integral. The main advantage of numerical procedures which use RBFs over traditional techniques is mesh-less property of these methods. RBFs are used actively for solving partial differential equations. The examples see [9-11]. In the last decade, the development of the RBFs as a truly meshless method for approximating the solutions of PDEs has drawn the attention of many researchers in science and engineering [12-14]. Meshless method has become an important numerical computation method, and there are many academic monographs are published [15-17].

The layout of the article is as follows: In section 2, we introduce the collocation method and apply this method on the convection-diffusion

${ }^{*}$ Corresponding author: Ling-De Su, Department of Mathematics, North-Eastern Federal University, Belinskogo, Yakutsk, Russia, Tel: +79676245017; E-mail: sulingde@gmail.com

Received February 10, 2015; Accepted April 29, 2015; Published May 08, 2015

Citation: Su LD, Jiang ZW, Jiang TS (2015) Numerical Method for OneDimensional Convection-diffusion Equation Using Radical Basis Functions. J Phys Math 6: 136. doi:10.4172/2090-0902.1000136

Copyright: (c) 2015 Su LD, et al. This is an open-access article distributed under the terms of the Creative Commons Attribution License, which permits unrestricted use, distribution, and reproduction in any medium, provided the original author and source are credited. 
equation. The results of numerical experiments are presented in section 3. Section 4 is dedicated to a brief conclusion. Finally, some references are introduced at the end.

\section{The Collocation Method with Radical Basis Function}

\section{Radial basis function approximation}

The approximation of a distribution $u(x)$, using RBF may be written as a linear combination of $\mathrm{N}$ radial functions, usually it takes the following form:

$$
u(\boldsymbol{x}) \approx \sum_{j=1}^{N} \lambda_{j} \phi\left(\boldsymbol{x}, \boldsymbol{x}_{j}\right)+\psi(\boldsymbol{x}), \text { for } \boldsymbol{x} \in \Omega \subseteq R^{d}
$$

Where $N$ is the number of data points, $x=\left(x_{1}, x_{2} \ldots x_{d}\right), d$ is the dimension of the problem, the $\lambda$ 's are coefficients to be determined and $\varphi$ is the radial basis function. Eq. (2.1.1) can be written without the polynomial $\psi$. In that case, $\varphi$ must be unconditional positive definite to guarantee the solvability of the resulting system (e. g. Gaussian or Inverse Multi quadrics). However, $\psi$ is usually required when $\varphi$ is conditionally positive definite, i. e, when $\varphi$ has a polynomial growth towards infinity. We will use the Multi quadrics (MQ), which defined as:

$$
\text { MQ: } \phi\left(\boldsymbol{x}, \boldsymbol{x}_{j}\right)=\phi\left(r_{j}\right)=\sqrt{r_{j}^{2}+c^{2}}, c>0
$$

Where $r_{j}=\left\|\boldsymbol{x}-\boldsymbol{x}_{j}\right\|$ is the Euclidean norm. Since $\varphi$ given by (2.2) is $\mathrm{C}^{\infty}$ continuous, we can use it directly.

If $\boldsymbol{P}_{q}^{d}$ denotes the space of $d$-variate polynomial of order not exceeding than $q$, and letting the polynomials $\left(P_{1}, P_{2}, \ldots, P_{m}\right)$ be the basis of $\boldsymbol{P}_{q}^{d}$ in $R^{d}$, then the polynomial $\psi(x)$ in Eq. (2.1) is usually written in the following form:

$$
\psi(\boldsymbol{x})=\sum_{i=1}^{m} \xi_{i} P_{i}\left(\boldsymbol{x}_{j}\right)
$$

Where $m=(q-1+d) ! /(d !(q-1) !)$. To get the coefficients $x=\left(\lambda_{1}, \lambda_{2} \ldots\right.$ $\left.\lambda_{N}\right)$, and $\left(\xi_{1}, \xi_{2}, \cdots, \xi_{m}\right)$, the collocation method is used. However, in addition to the $N$ equations resulting from collecting Eq. (2.1.1) at $N$ points and extra $m$ equations are required. This is ensured by the $m$ conditions for Eq. (2.1),

$$
\sum_{j=1}^{N} \lambda_{j} P_{i}\left(\boldsymbol{x}_{j}\right)=0, i=1,2, \cdots m .
$$

In a similar representation as Eq. (2.1), for any linear partial differential operator $\ell, \ell \boldsymbol{u}$ can be approximated by:

$$
\ell u(x) \approx \sum_{j=1}^{N} \lambda_{j} \ell \phi\left(x, x_{j}\right)+\ell \psi(x) .
$$

\section{The convection-diffusion equation}

Let us consider the 1D convection-diffusion equation Eq. (1.1), with the initial conditions Eq. (1.2) and the Dirichlet boundary conditions Eq. (1.3).

First, let us discretize Eq. (1.1) according to the following $\theta$-weighted scheme:

$$
\begin{aligned}
& \frac{u(x, t+\tau)-u(x, t)}{\tau}+\alpha \cdot \nabla u(x, t)= \\
& \theta\left(\varepsilon \cdot \nabla^{2} u(x, t+\tau)-\beta \cdot u(x, t+\tau)\right)+(1-\theta)\left(\varepsilon \cdot \nabla^{2} u(x, t)-\beta \cdot u(x, t)\right)+f(x, t)
\end{aligned}
$$

where $0 \leq \theta \leq 1$, and $\tau$ is the time step size, and $\nabla u=\frac{\partial u}{\partial x}$, using the notation $u^{n}=u\left(x, t^{n}\right)$ where $t^{n}=t^{n-1}+\tau$, we get:

$$
\begin{aligned}
& (1+\theta \cdot \beta \cdot \tau) \cdot u^{n+1}-\theta \cdot \tau \cdot \varepsilon \cdot \nabla^{2} u^{n+1}= \\
& (1-(1-\theta) \cdot \beta \cdot \tau) \cdot u^{n}+(1-\theta) \cdot \tau \cdot \varepsilon \cdot \nabla^{2} u^{n}-\alpha \cdot \tau \cdot \nabla u^{n}+\tau \cdot f^{n}
\end{aligned} .
$$

Assuming that there are $N-2$ interpolation points, $u^{n}(x)$ can be approximated by:

$$
u^{n}(x) \approx \sum_{j=1}^{N-2} \lambda_{j}^{n} \phi\left(r_{j}\right), j=1,2, \cdots, N-2 .
$$

To guarantee the positive definition, here we use the following approximation:

$$
u^{n}\left(x_{i}\right) \approx \sum_{j=1}^{N-2} \lambda_{j}^{n} \phi\left(r_{i j}\right)+\lambda_{N-1}^{n} x_{i}+\lambda_{N}^{n},
$$

Where $r_{j}=\left\|\boldsymbol{x}-\boldsymbol{x}_{j}\right\|$ is the Euclidean norm. The additional conditions due to Eq. (2.4) are written as:

$$
\sum_{j=1}^{N-2} \lambda_{j}^{n}=\sum_{j=1}^{N-2} \lambda_{j}^{n} x_{j}=0 .
$$

Writing Eq. (2.9) together with Eq. (2.10) in a matrix form we have:

$$
[u]^{n}=A[\lambda]^{n},
$$

Where $[u]^{n}=\left[\begin{array}{llllll}u_{1}^{n} & u_{2}^{n} & \cdots & u_{N-2}^{n} & 0 & 0\end{array}\right]^{T}, \quad[\lambda]^{n}=\left[\begin{array}{llll}\lambda_{1}^{n} & \lambda_{2}^{n} & \cdots & \lambda_{N}^{n}\end{array}\right]$ and $A=\left[a_{i j}, \quad 1 \leq i, j \leq N\right]$ is given by:

$$
A=\left(\begin{array}{ccccc}
\phi_{11} & \cdots & \phi_{1(N-2)} & x_{1} & 1 \\
\vdots & \ddots & \vdots & \vdots & \vdots \\
\phi_{(N-2) 1} & \cdots & \phi_{(N-2)(N-2)} & x_{N-2} & 1 \\
x_{1} & \cdots & x_{N-2} & 0 & 0 \\
1 & \cdots & 1 & 0 & 0
\end{array}\right) .
$$

Assuming that there are $p<N-2$ internal points and $N-2-p$ boundary points, then the $N \times N$ matrix $A$ can be split into: $A=A_{d}+A_{b}+A_{e}$, where

$$
\begin{aligned}
& A_{d}=\left[\begin{array}{lllll}
a_{i j} & \text { for } & (1 \leq i \leq p, 1 \leq j \leq N) \quad \text { and } & 0 & \text { elsewhere }
\end{array}\right] \\
& A_{b}=\left[\begin{array}{lllll}
a_{i j} & \text { for } & (p+1 \leq i \leq N-2,1 \leq j \leq N) \quad \text { and } & 0 & \text { elsewhere }
\end{array}\right] \\
& A_{e}=\left[\begin{array}{lllll}
a_{i j} & \text { for } & (N-1 \leq i \leq N, 1 \leq j \leq N) \text { and } 0 & \text { elsewhere }
\end{array}\right]
\end{aligned}
$$

Using the notation $\ell A$ to designate the matrix of the same dimension as $A$ and containing the elements $\hat{a}_{i j}$ where $\hat{a}_{i j}=\ell a_{i j}$, $1 \leq i, j \leq N$, then Eq. (2.2.1) together with the boundary conditions Eq. (1.3) can be written in matrix form as:

$B \lambda^{n+1}=C \lambda^{n}+\tau \cdot f^{n}+[G]^{n+1}$,

where

$$
\begin{aligned}
& C=(1-\beta(1-\theta) \tau) A_{d}+(1-\theta) \tau \varepsilon \nabla^{2} A_{d}-\alpha \tau \nabla A_{d}, \\
& B=(1+\beta \theta \tau) A_{d}-\theta \tau \varepsilon \nabla^{2} A_{d}+A_{b}+A_{e}, \\
& {[G]^{n+1}=\left[\begin{array}{llllllll}
0 & \cdots & 0 & g_{p+1}^{n+1} & \cdots & g_{N-2}^{n+1} & 0 & 0
\end{array}\right]^{T},} \\
& {[f]^{n}=\left[\begin{array}{llllll}
f_{1}^{n} & \cdots & f_{p}^{n} & 0 & \cdots & 0
\end{array}\right]^{T} \text {. }}
\end{aligned}
$$

Eq. (2.14) is obtained by combining Eq. (2.6), which applies to the 
Citation: Su LD, Jiang ZW, Jiang TS (2015) Numerical Method for One-Dimensional Convection-diffusion Equation Using Radical Basis Functions. J Phys Math 6: 136. doi:10.4172/2090-0902.1000136

Page 3 of 5

domain points, while Eq. (1.3) applies to the boundary points. Together with the initial condition Eq. (1.2) and Eq. (2.14), we can get all $\lambda$ 's, thus we can get the numerical solutions.

Since the coefficient matrix is unchanged in time steps, we use the $L U$ factorization to the coefficient matrix only once and use this factorization in our algorithm.

Remark: Although Eq. (2.14) is valid for any value of $\theta \in[0,1]$, we will use $\theta=\frac{1}{2}$ (The famous Crank-Nicolson scheme).

\section{Numerical Examples}

In this section, we present several numerical results to confirm the efficiency of our algorithm for solving the 1D convection-diffusion equation.

\section{Example 1}

In this example, we consider the convection-diffusion Eq. (1.1) in $[0,1]$ with $\alpha=0.1, \beta=0, \varepsilon=0.01$, with the boundary conditions:

$u(0, t)=\exp (0.11 t), \quad u(1, t)=\exp (-1+0.11 t), \quad t>0$,

And the initial condition

$u(x, 0)=\exp (-x) \quad 0 \leq \mathrm{x}, \mathrm{y} \leq 1$,

Then the analytical solution of the equation is $u(x, t)=\exp (-x+0.11 t)$. The right side functions $f(x, t)=0$.

We use $M Q$ radical basis function for the computation, the $L_{\infty}, L_{2}$ and RMS errors and Root-Mean-Square (RMS) of errors are obtained in Table 1 for $\mathrm{t}=0.1,0.3,0.5,0.7$ and 1.0 with time steps $\tau=0.001$ and $d x=0.001$.

The space-time graph of analytical and numerical solutions for $t=1$ are given in Figure 1 . Note that we cannot distinguish the exact solution from the estimated solution in Figure 1.

\begin{tabular}{|l|c|c|c|c|c|}
\hline $\mathbf{T}$ & $\mathbf{0 . 1}$ & $\mathbf{0 . 3}$ & $\mathbf{0 . 5}$ & $\mathbf{0 . 7}$ & $\mathbf{1 . 0}$ \\
\hline $\mathbf{L}_{\infty}$-errors & $1.547 \times 10^{-5}$ & $2.371 \times 10^{-4}$ & $3.599 \times 10^{-4}$ & $4.639 \times 10^{-4}$ & $5.909 \times 10^{-4}$ \\
\hline $\mathbf{L}_{2}$-errors & $6.788 \times 10^{-5}$ & $1.787 \times 10^{-4}$ & $3.453 \times 10^{-4}$ & $3.453 \times 10^{-4}$ & $4.372 \times 10^{-4}$ \\
\hline RMS- errors & $6.778 \times 10^{-5}$ & $1.784 \times 10^{-4}$ & $3.448 \times 10^{-4}$ & $3.448 \times 10^{-4}$ & $4.365 \times 10^{-4}$ \\
\hline
\end{tabular}

Table 1: For $\mathrm{T}=0.1,0.3,0.5,0.7$ and 1.0 with time steps $\mathrm{T}=0.001$ and $d x=0.001$.

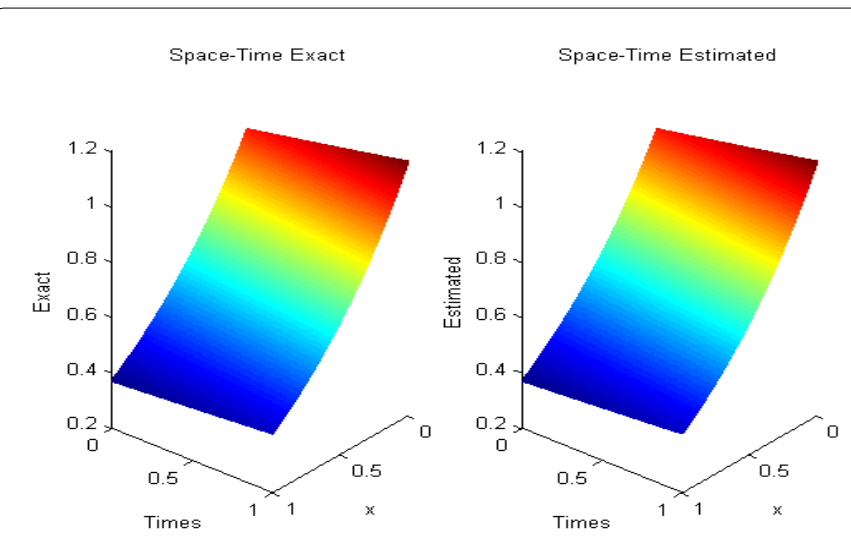

Figure 1: Space-time graph of the exact and estimated solutions with $T=1$ for Example 1.

\begin{tabular}{|l|c|c|c|c|c|}
\hline $\mathbf{T}$ & $\mathbf{0 . 1}$ & $\mathbf{0 . 2 5}$ & $\mathbf{0 . 5}$ & $\mathbf{0 . 7 5}$ & $\mathbf{1 . 0}$ \\
\hline $\mathbf{L}_{\infty}$-errors & $8.855 \times 10^{-5}$ & $1.031 \times 10^{-4}$ & $1.245 \times 10^{-4}$ & $1.501 \times 10^{-4}$ & $1.811 \times 10^{-4}$ \\
\hline $\mathbf{L}_{\mathbf{2}}$-errors & $5.929 \times 10^{-5}$ & $6.882 \times 10^{-5}$ & $8.305 \times 10^{-5}$ & $1.002 \times 10^{-4}$ & $1.208 \times 10^{-4}$ \\
\hline $\mathbf{R M S}$ - errors & $5.842 \times 10^{-5}$ & $6.781 \times 10^{-5}$ & $8.183 \times 10^{-5}$ & $9.870 \times 10^{-5}$ & $1.191 \times 10^{-4}$ \\
\hline
\end{tabular}

Table 2: Numerical Errors at different times with $T=0.001$ and $d x=0.001$ for Example 2.

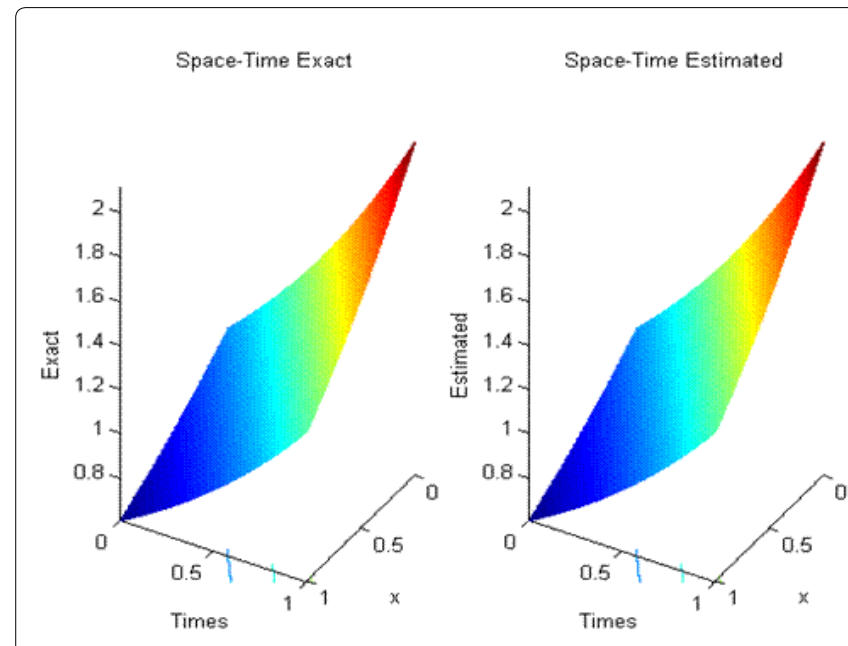

Figure 2: The space-time graph of exact and numerical solutions with $\mathrm{T}=1$ for Example 2.

\section{Example 2}

In this example, we consider Eq. (1.1) with $\alpha=1, \beta=0, \varepsilon=1$ and the boundary conditions:

$$
u(0, t)=\exp \left(\frac{3 t}{4}\right), u(1, t)=\exp \left(\frac{-2+3 t}{4}\right)
$$

And the analytical solution of the equation is given as:

$$
u(\mathrm{x}, t)=\exp \left(\frac{-2 x+3 t}{4}\right)
$$

We get the initial conditions from the exact solution. The right side functions $f(x, t)=0$.

The $L_{\infty}, L_{2}$ and RMS errors and Root-Mean-Square (RMS) of errors are obtained in Table 2 for $\mathrm{T}=0.1,0.25,0.5,0.75$ and 1.0 with time steps $\tau=0.001$ and $d x \quad 0.01$.

Similar to the previous example, the space-time graph of analytical and estimated solutions for $\mathrm{t}=1$ are presented in Figure 2 .

\section{Example 3}

We consider the convection-diffusion equation Eq. (1.1) with $\alpha=-1, \beta=10$ and $\varepsilon=1$ in the interval $[0,1]$, the exact solution is given as $u(x, t)=t \sin (\pi x) \exp \left(-\pi^{2} t\right)$. The boundary conditions are:

$$
u(0, t)=0, u(1, t)=0, \mathrm{t} \geq 0,
$$

The right side functions of $f(x, t)=[(1+10 t) \sin (\pi x)-\pi t \cos (\pi x)] \exp \left(-\pi^{2} t\right)$, and we extract the initial conditions from the exact solution.

These results are obtained with $d x=0.001, \tau=0.001$. Similar to the previous examples, the $L_{\infty}$ and $L_{2}$ error and RMS errors for $\mathrm{t}=0.5$, $0.75,1.0,1.25$ and 1.5 are presented in Table 3 . 
Citation: Su LD, Jiang ZW, Jiang TS (2015) Numerical Method for One-Dimensional Convection-diffusion Equation Using Radical Basis Functions. J Phys Math 6: 136. doi:10.4172/2090-0902.1000136

\begin{tabular}{|l|c|c|c|c|c|}
\hline $\mathbf{T}$ & $\mathbf{0 . 5}$ & $\mathbf{0 . 7 5}$ & $\mathbf{1 . 0}$ & $\mathbf{1 . 2 5}$ & $\mathbf{1 . 5}$ \\
\hline $\mathbf{L}_{\infty}$-errors & $1.547 \times 10^{-5}$ & $2.112 \times 10^{-6}$ & $2.472 \times 10^{-7}$ & $2.673 \times 10^{-8}$ & $2.757 \times 10^{-9}$ \\
\hline $\mathbf{L}_{\mathbf{2}}$-errors & $1.179 \times 10^{-5}$ & $1.595 \times 10^{-6}$ & $1.859 \times 10^{-7}$ & $2.007 \times 10^{-8}$ & $2.066 \times 10^{-9}$ \\
\hline RMS- errors & $1.177 \times 10^{-5}$ & $1.593 \times 10^{-6}$ & $1.856 \times 10^{-7}$ & $2.104 \times 10^{-8}$ & $2.063 \times 10^{-9}$ \\
\hline
\end{tabular}

Table 3: Numerical Errors at different times with $T=0.001$ and $d x=0.001$ for Example 3.

Space-Time Exact

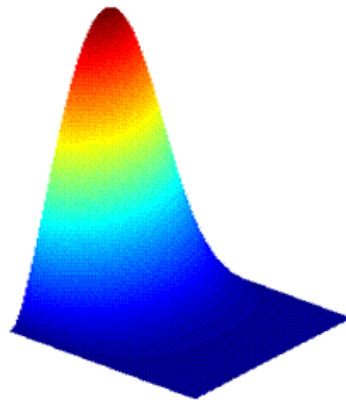

Figure 3: Space-time graph of the exact and estimated solutions with $T=1$ for Example 3.

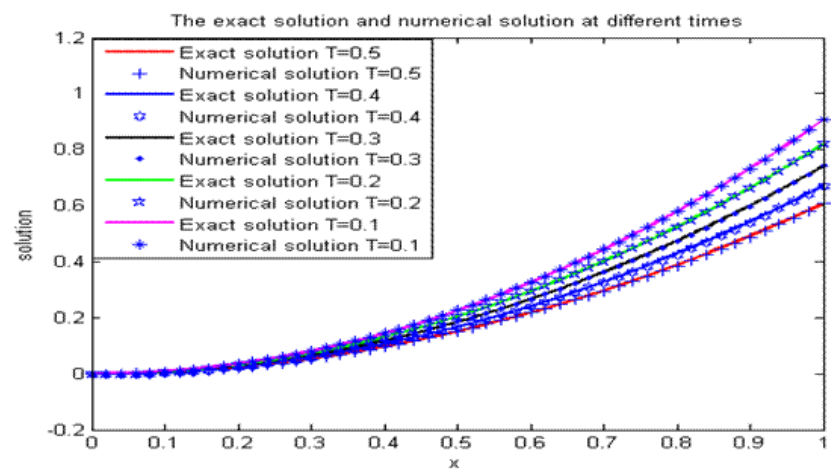

Figure 4: The exact and estimated solutions at different times for Example 4.

The space-time graph of analytical and numerical solution for $\mathrm{t}=1$ is presented in Figure 3.

\section{Example 4}

In this example, we consider the convection-diffusion equation in $[0,1]$ has the following form:

$$
\frac{\partial u}{\partial t}+\frac{\partial u}{\partial x}+2 u=\frac{\partial^{2} u}{\partial^{2} x}+f(x, t)
$$

The right side functions $f(x, t)=\left(x^{2}+2 x-2\right) \exp (-t)$, with the boundary condition:

$$
u(0, t)=0, u(1, t)=\exp (-t), \mathrm{t}>0
$$

Then the analytical solution of the equation is $u(x, t)=x^{2} \exp (-t)$, we get the initial conditions from the exact solution.

In this case, we use the radial basis functions MQ for the discussed scheme. These results are obtained for $d x=0.001, \tau=0.001$. The graph of analytical and numerical solution for $\mathrm{t}=0.1,0.2,0.3,0.4$ and 0.5

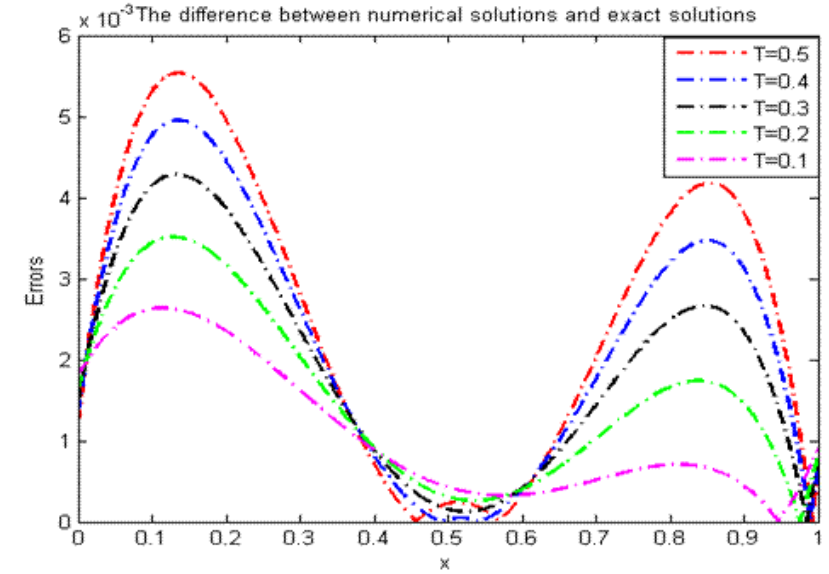

Figure 5: The exact and estimated solutions at different times for Example 4.

is given in Figure 4. The results obtained show the very good accuracy and efficiency of the new approximate scheme. Note that we cannot distinguish the exact solution from the estimated solution in Figure 4.

We also give the difference between exact solutions and numerical solutions in Figure 5.

\section{Conclusion}

In this paper, the collocation method is employed for the numerical solution of convection-diffusion equation based on radical basis functions (RBFs). Coupled with the time discretization, the proposed method is a truly meshless method which requires neither domain nor boundary discretization. The results of numerical experiments are presented, and are compared with analytical solutions confirmed the good accuracy of the presented scheme.

\section{Acknowledgement}

This work was supported by the national Natural Science Foundation of China (Grant Nos.11301252, 11201212) and Applied Mathematics Enhancement Program of Linyi University.

\section{References}

1. Kurganov A, Tadmory E (2000) A New High-Resolution Central Schemes for Nonlinear Conservation Laws and Convection-Diffusion Equations. Journal of Computational Physics 160: 369-383.

2. Stocker T (2014) Introduction to Climate Modelling [M]. Springer.

3. Scott A, Socolofsky, Jirka GH Advective Diffusion Equation.

4. Cockburn B, Shu CW (1998) The Local Discontinuous Galerkin Method for Time-Dependent Convection-Diffusion Systems. Siam J Numer Anal 35: 24402463.

5. Douglas J, Russell TF (1982) Numerical Methods for Convection-Dominated Diffusion Problems Based on Combining the Method of Characteristics with Finite Element or Finite Difference Procedures. Siam J Numer Anal 19: 871885.

6. Chen W, Hon YC (2003) Numerical investigation on convergence of boundary knot method in the analysis of homogeneous Helmholtz, modified Helmholtz and convection-diffusion problems. Int J Numer Meth Engng 56: 1931-1948.

7. Lin H, Atluri SN (2000) Meshless Local Petrov-Galerkin (MLPG) Method for Convection-Diffusion Problems. Computer Modeling in Engineering \& Sciences 1: $45-60$.

8. Manohar RP, Stephenson JW (1984) A single cell high order scheme for the convection-diffusion equations with variable coefficients. Int $\mathrm{J}$ for Num Meth in Fluids 4: 641-651. 
Citation: Su LD, Jiang ZW, Jiang TS (2015) Numerical Method for One-Dimensional Convection-diffusion Equation Using Radical Basis Functions. J Phys Math 6: 136. doi:10.4172/2090-0902.1000136

Page 5 of 5

9. Jiang ZW, Su LD, Jiang TS (2014) A Meshfree Method for Numerical Solution of Nonhomogeneous Time-Dependent Problems. Abstract and Applied Analysis.

10. Kansa EJ (1990) Multiquadrics-a scattered data approximation scheme with applications to computational fluid dynamics-I. Comout Math Appl 19: 127-145.

11. Zerroikat M, Power H, Chen CS (1992) A numerical method for heat transfer problem using collocation and radial basis functions. Int I Numer Meth Eng 42: 1263-1278.

12. Li M, Jiang TS, Hon YC (2010) A meshless method based on RBFs method for nonhomogeneous backward heat conduction problem. Engineering Analysis with Boundary Elements 34: 785-792.

13. Su LD, Jiang ZW, Jiang TS (2013) Numerical solution for a kind of nonlinear telegraph equations using radial basis functions. Communications in Computer and Information Science 391: 140-149.

14. Jiang TS, Li M, Chen CS (2012) The Method of Particular Solutions for Solving Inverse Problems of a Nonhomogeneous Convection-Diffusion Equation with Variable Coefficients. Numerical Heat Transfer, Part A: Applications 61: 338-352.

15. Fasshauer GE (2008) Meshfree Approximation Methods with MATLAB [M] Illinois Institute of Technology.

16. Liu GR, Gu YT (2005) An introduction to meshfree methods and there programming $[\mathrm{M}]$. Springer.

17. Chen W, Fu ZJ, Chen CS (2014) Recent Advances in Radial Basis Function Collocation Methods [M]. Springer Briefs in Applied Sciences and Technology. 